Research Journal of Applied Sciences, Engineering and Technology 6(12): 2233-2237, 2013

DOI:10.19026/rjaset.6.3852

ISSN: 2040-7459; e-ISSN: 2040-7467

(C) 2013 Maxwell Scientific Publication Corp.

\begin{tabular}{lll}
\hline Submitted: December 12, 2012 & Accepted: February 08, 2013 & Published: July 30, 2013
\end{tabular}

Research Article

\title{
A Brief Review on Inspection Methods of High Pressure Vessels Applied in Food Processing
}

\author{
${ }^{1,2}$ Rong-Li Li, ${ }^{2}$ Xiao-Yong Liu, ${ }^{1}$ Shou-Qin Zhang, ${ }^{3}$ Tao Li and ${ }^{3}$ Guo-Jing Ren \\ ${ }^{1}$ College of Biological and Agricultural Engineering, Jilin University, Changchun 130022, China \\ ${ }^{2}$ College of Mechanical Engineering, Beihua University, Jilin 132021, China \\ ${ }^{3}$ China Electronics Technology Group Corporation No.49 Research Institute, Harbin 150001, China
}

\begin{abstract}
In this review, inspection methods used in the high pressure vessel in-service, such as strain electrical measuring method, eddy current testing, ultrasonic testing, acoustic emission and optical measurement methods were summarized. The principles, characters and research progress of the above mentions inspection methods when they are used for inspecting HPV were reviewed. Finally, an attempt is made to look into the future of inspection methods of HPV with personal opinions.
\end{abstract}

Keywords: DSCM, HPV, NDT, optical measurement method

\section{INTRODUCTION}

The High Pressure Vessel (HPV) is widely used in chemical, petrochemical industry, food process fields and so on. The HPV employed in food process particularly specializes in quick-actuating, high working pressure and long fatigue life (Ma, 2006). Therefore, it is necessary to measure the various mechanical properties and behaviors of the HPV by various methods to ensure the quality of product and safety running in use. Currently, the inspections of the HPV are consisted of three parts, that is, raw material inspection, fabrication inspection and in-service inspection (Shen and Zheng, 2004). There are many raw material and inspection methods, such as ultrasonic, radiographic and eddy current. The fabrication inspection methods are magnetic particle, penetrant and acoustic emission, exclude above methods. In this study, some advanced testing technologies will be brief reviewed to measure the deformation information of the HPV in-service. The focuses will be on the surface inspection of the HPV.

\section{INSPCTION METHODS AND APPLICATIONS OF THE HPV IN-SERVICE}

It is a big trouble to detect inner surface defects by some methods, such as radiographic testing, etc., because ratio between inner diameter and outsider diameter of the HPV is usually less than 0.6. The primary in-service inspection methods are magnetic particle testing, metal magnetic memory testing, industrial inner peek-mirror examination, penetrat testing, eddy current testing and so on. The ultrasonic testing can examine both the inner defects and the inner surface defects. Acoustic emission testing was a realtime monitor of the pressure vessel during its operation (Yue et al., 1995).

Strain electrical measuring method: It is a classical testing method to measure strains on the HPV's surface by strain gauges. Inhere advantages are measuring strains of the high precision, fast response for widely applying in stress and strain measurements in the HPV. But it wasn't obtained full field stress information (Zhou and Shen, 2005; Sposito et al., 2010). Ma (2006) and Zhang (2005) had completed stress and strain measurement on the cylinder and stopper of the HPV employed in seafood process and a new type of the HPV, respectively. Ma (2006) had tested 33 points to analyze the stress distribution on the cylinder and flange at different pressures. Although the correct results were obtained by testing, it was difficult to obtain full-field deformation information by this method. Taylor et al. (2012) employed the neutron diffraction and mechanical slitting technique to determine the residual stress state of the clad steel cylinders. Both techniques provided triaxial stress data and useful information on the effects of explosive cladding. The former was attractive because it was nondestructive; Slitting, although destructive, produced the more representative results for the whole sample and obtained more accurate the slitting results.

Eddy current testing: Eddy current Testing (ET) (Li and Shen, 2004) is a Nondestructive Testing (NDT) 
method that measures conductive material surface and near-surface defects based on the principle of electromagnetic induction. It is difficult to distinguish between changes in the signal due to creep damage and those deriving from other sources. There are many advantages for eddy current testing, such as rapid testing, higher sensitivity, evaluation for different sizes of the defects, special-shaped material and small parts detection at higher temperature, etc. But it only defects conductivity material and couldn't judge defects' types. Recently, Eddy current testing is used to inspect welding cracks by a magneto probe based on the electric current perturbation method. It is fit for fast cracks testing and cracks depth measuring of large area metal parts and rough surface metal parts. It is effective when it is used in rapid cracks testing and cracks depth measuring of the HPV. The superiority of the special inspection lies on its inner or outsider inspection of the HPV. He et al. (2009) cited that Hatsukade detected fatigue of filament wound composite pressure vessel with aluminum liner by eddy current inspection based on quantum interference devices. The results stated that there were all-over part-through cracks in the aluminum liner on elliptical closure of pressure vessel under cyclic pressure.

Ultrasonic testing: Ultrasonic Testing (UT) (Pan et al., 2004) is a NDT method, which to detect defects by ultrasound reflected when meets objects and attenuates during transmission in the medium. Its advantages are high sensitivity, good directionality, strong ability to penetrate and fast inspection speed. Owing to its small volume, light weight, easy-to-carry, it is widely used in the UHPV inspection. UT can examine inner surface defects of the UHPV from outside by transverse waves. The longitudinal wave is used to inspect cracks of buttress thread on the stopper of the UHPV. In addition, UT is not accurate for qualitative and quantitative defects. Bray (2002) detected changes in wall and weld stress by the $\mathrm{L}_{\mathrm{CR}}$ ultrasonic technique. At low wall stresses, the ultrasonic data showed considerable scatter. There was similar scatter in the zero pressure travel-times at individual locations around the vessel. At higher wall stresses, there was an almost linear relationship of stress and travel-time change. Knapp (Yao et al., 2005) gave a new concept for monitoring the structural integrity of filament wound pressure vessel using an optical sensor. Guo et al. (2006) studied circumferential ultrasonic testing for UHPVs and pipes, presented parameters for radial defects detection with circumferential ultrasonic testing based on theoretical as well as experimental investigations on the structure of sound field, diversity comparisons of radial defects echo, sensitivity of surface radial defects, defect location and sensitivity adjustment of the defects in thick-walled cylinder. Field tests of ultra-high pressure vessels for man-made crystal production and ultra-high pressure pipes for polyethylene showed that the presented methods had sufficient sensitivity and reliability for detecting radial defects.

Acoustic emission: Acoustic Emission (AE) technique is called a dynamic test because it monitors changes in configurations caused by imposed structural stress. The technique relies on the transient sound wave, which is generated by fracture in and then propagating through the tested material. AE has many advantages, such as its continuous and in-situ monitoring capability to examine active defects and damages in loading structures ( $\mathrm{Li}$ et al., 2010). Therefore, AE technique has been applied to many fields, including pressure vessel. $\mathrm{AE}$ monitoring of pressure vessels has more than 20 years' history. Ohtsuka et al. (1981) monitored on the model vessel of low carbon steel containing several kinds of artificial defects and on simulating specimens in laboratory tests by AE. Cracks, leaks and leak conditions in pressure vessels were obtained by the size of the AE signals (Rastogi, 1997). Nokes (Yao et al., 2005) studied deformations of composite pressure vessels using the AE. Ennaceur et al. (2006) obtained the correlation between $\mathrm{AE}$, the method of potential difference and microfractographic observation. He might predict the remaining service life of a vessel from the results of short-term AE monitoring of crack propagation. It was used to monitor fatigue cracks in pressure pipe under cyclic pressure by Ai et al. (2010). It was found that the changes of real time $\mathrm{AE}$ was sensitive to movement of microscopic structure. The amplitude and energy showed activity of fatigue crack well. The differential distribution of amplitude together with cumulative event number not only revealed trend of active defect well but also gave out some hint of potential leak in piping. Máthis et al. (2011) studied strain rate tensile tests of stainless steel in supercritical water environment by the AE. Laksimi et al. (2012) studied the behavior of weld defects in pressure equipment by $\mathrm{AE}$ and showed that $\mathrm{AE}$ parameters representative of a weld defect in a tank could to predict of premature failures of pressure equipment.

\section{OPTICAL MEASUREMENT METHODS}

Optical measurement methods are kinds of inspection methods based on optical interference or as the foundation of the principle of digital image analysis technique. These methods are divided into classical and modern optical methods by laser emerging in the 1960's and digital image processing technology's maturity. Only the photoelastic can obtain stress distribution of model, other methods belong to deformation measurement method (Rastogi, 1997). Because the HPV employed in food processing is often working under high-pressure or ultra-high-pressure condition, its deformation and failure process with more complex and serious heterogeneity stress are generated on the 
UHPV's cylinder at different radius during pressurizing. Compared with conventional measurement methods, the Digital Speckle Correlation Metrology (DSCM) is better to obtain full field information of the HPV inspection. Most optical detection methods can accomplish non-contact measurement and get whole-field information. So it is an important mean of stress analysis to detect the HPV.

Moiré interferometry method: Moiré interferometry method belongs to a new NDT method detected displacement field and strain field of surfaces by the moiré patterns. The principle of this method is as follows: The deformations information of a specimen's surface is carried by a grating called a model grating. This grating acts as a reference element of the changes of the surface from the reference state to the deformed state. These changes reveal the relative deformations of the specimen surface. To obtain the changes in the geometry of the model grating, a master grating is introduced. The superposition of the two gratings produces the moiré patterns. These patterns are shown on the observation system. It is necessary to acquire the relationship among the master grating, the model grating, the observation system and the specimen surface to determine displacements and strains. This relationship depends on the particular technique utilized. There are three particular techniques utilized, such as intrinsic-moiré method, projection moiré method and reflection moiré method. It is especially suitable to large strain and large deformation measurement.

In the past few years, the moiré method has been extensively used in fracture mechanics, particularly in the study of the plastic region in the neighborhood of the crack tip, to analyze ductile fracture and to study fatigue crack growth. An industrial application to the test of a large PV in the plastic range was described by Sciammarella (1982). Huang et al. (1986) studied the displacement field and the strain field of the fatigue crack tip on the HPV by the moiré interferometry method. Liu et al. (1991) measured the displacement field, the strain field, $\mathrm{K}$ and COD of the axial through crack tip of HPV. Sha and Chen (1994) determined the elastic-plastic strain, the size of the plastic zone and COD date around the crack tip on the HPV by the moiré method. The experiments results were agreed well with those calculated by the theory formulation and the four kinds of COD design curves.

The displacement measurement sensitivity is determined by frequency of grating, the higher the frequency of grating, the higher the sensitivity. It is higher sensitivity to consider virtual grating used in laser interference as reference grating. But its application in the UHPV is also limited owing to sticking films on the surface of loaded objects, using lasers and special lights.

Digital speckle correlation metrology: As one of the advanced experimental stress analysis technologies,

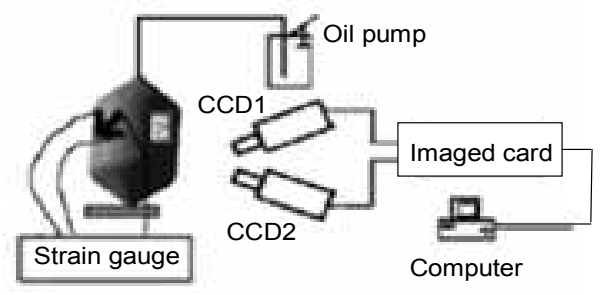

Fig. 1: Test system on composite vessel. Reproduced with permission by Meng (2006), copyright 2006, elsevier

Digital Speckle Correlation Metrology (DSCM), also known as Digital Image Correlation (DIC), is widely applied in deformation measurements and nondestructive testing. The DSCM was first conceived and developed by Peter and Ranson at the University of South Carolina in the early 1980s. The DSCM is a whole-field image analysis method based on grey value digital images, which can determine the contour and the displacements of an object under loads. The grey values of the object before and after deformation/ displacement/strain are obtained by the DSCM and then DSCM is used to calculate the correlation coefficient changes and found out the maximum correlation coefficient values by iteration method to measure the corresponding deformation, displacement and strain. The studies of DSCM are from characteristics of the interference field and quality of the fringe pattern to characteristics of the interference field and the theory of speckle correlation metrology in optical manner (Meng et al., 2006a).

Because it only needs to acquire images of a deformed or moved object, which are then analyzed to extract full-field displacement and the shape information. There is not complex relationship between the deformation and the used optical system and the characteristic pattern on the tested object surface, such as lines, grids, dots and random arrays. Therefore, DSCM has been widely applied in many areas of science and engineering measurements.

With the development of CCD technology and computer vision technology, DSCM measurements have developed from two-dimensional to threedimensional. DSCM is used to measure 3D surface shape and deformations by a variety of illumination sources for a wide range of material systems. Luo and Chao first presented 3D displacement measurement from a stereo pair of CCD cameras in 1993. The fullfield deformation measurement of carbon fiber/epoxy composite pressure vessel was investigated using DSCM by Yao et al. (2005). The full-field displacement and strain distribution of a composite pressure vessel were evaluated under internal pressure.

Three-dimensional structure of DSCM signal detection system is shown in Fig. 1, it requires two or more cameras capture images. DSCM technique can employ white light illumination, but it requires 
existence of speckle textures by artificial on the sample surface. The 3D full-field deformation of a composite pressure vessel was studied using a 3D DSCM by Meng et al. (2006b). The 3D full field displacement and strain distribution of the composite pressure vessel were evaluated under internal pressure loading. The average strain value showed good agreement with the results obtained from conventional electrical-resistance strain gauge technology. The results revealed that the 3D DSCM was suitable for deformation measurement of composite pressure vessels in real engineering practice.

Properties of different materials, fatigue and breakup of different materials, even for hightemperature objects are measured by DSCM technology, among which the measurement of strain and flaw can provide rich information for NDT. It also can measure objects, which size scales ranging from meters to micrometers or nanometers. Even the interior deformations can be measured through volumetric imaging by use of such a technology as computer tomography, while the dynamic/impact behaviors of materials can also be investigated with a high-speed camera system.

Prospects: With the development of computer science, material and image processing, many new techniques and applications have been discovered and exploited, which enrich strongly the available methods, techniques and systems of detecting of HPV. However, there are still many needs for its further improvement in various aspects, such as miniaturization of instruments by NDT, a wide detecting range measurement, technology for special and extreme environments and novel methods, techniques and systems in NDT. The DSCM applied for inspection of HPV will have an amazing future by personal opinions.

\section{DISCUSSION AND CONCLUSION}

All kinds of NDT applied in HPV or UHPV were outlined and briefly reviewed in this study. Optical methods had shown its special merits in deformation measurement of structures, such as non-contact, simple optical setup, no special preparation of specimens and no special requirement for the test environment, no sensitivity to the illumination and vibration. Therefore, it was the most suitable technique to measure the deformations of the HPV or their components when these devices or components were subjected to thermal or external loading. Then the prospects of the HPV' measurement were proposed.

\section{REFERENCES}

Ai, Q., C.X. Liu, X.R. Chen, P. He and Y. Wang, 2010. Acoustic emission of fatigue crack in pressure pipe under cyclic pressure. Nucl. Eng. Des., 240: 3616-3620.
Bray, D.E., 2002. Ultrasonic stress measurement and material characterization in pressure vessels, piping and welds. Proceeding of the Transactions of the ASME, 124: 326-335.

Ennaceur, C., A. Laksimia, C. Herve' and M. Cherfaoui, 2006. Monitoring crack growth in pressure vessel steels by the acoustic emission technique and the method of potential difference. Int. J. Pressure Vessel Piping, 83(3): 197-204.

Guo, W.C., J.Y. Zheng, X.F. Yu, X.B. Fang, H. Wang and Q.J. Qian, 2006. Circumferential ultrasonic testing for ultra-high pressure vessels and pipes (1). 23(10): 9-13, (In Chinese).

He, X.D., J.Q. Zhao, R.G. Wang and W.B. Liu, 2009. Review on nondestructive inspection of composite pressure vessel. J. Harbin Instit. Technol., 41(12): 78-82, (In Chinese).

Huang, X.K., J.Z. Pan and D.Y. Ju, 1986. The strain field of the fatigue crack tip on the HPV by the moiré interferometry method. Pressure Vessel Technol., 3(2): 25-31, (In Chinese).

Laksimi, A., C. Herve, C. Ennaceur and M. Cherfaoui, 2012. AE study of damage evolution in pressure vessels under cyclic loading. Proceedings of the 30th European Conference on Acoustic Emission Testing, pp: 1-12.

Li, X.T. and G.T. Shen, 2004. Nondestructive testing of pressure vessels: Eddy current testing technique. NDT, 26(8): 411- 430, (In Chinese).

Li, X.D., H.M. Xie, Y.L. Kang and X.P. Wu, 2010. A brief review and prospect of experimental solid mechanics in China. Acta Mech. Solid. Sinica, 23(6): 498-548.

Liu, K.L., B.Z. Pan, W.J. Wang and N.L. Zhao, 1991. A study on the sticking film moire interferometry for the crack of pressure vessel. J. Daqing Petrol. Instit., 15(4): 49-55, (In Chinese).

Ma, X., 2006. Research on key technology of self protective ultra high pressure vessel with toothlocked quick-actuating closure used in seafood processing. Ph.D. Thesis, Zhejiang University, pp: 61-159, (In Chinese).

Máthis, K., D. Prchal, R. Novotny' and P. Hahner, 2011. Acoustic emission monitoring of slow strain rate tensile tests of 304L stainless steel in supercritical water environment. Corros. Sci., 53: 59-63.

Meng, L.B., G.C. Jin, X.F. Yao and M. Luo, 2006a. The digital speckle correlation method and the application in deformation measurement of carbon fiber composite pressure vessel. Opt. Tech., 32(2): 163-168, (In Chinese).

Meng, L.B., G.C. Jin, X.F. Yao and H.Y. Yeh, 2006b. 3D full-field deformation monitoring of fiber composite pressure vessel using 3D digital speckle correlation method. Polym. Test., 25: 42-48. 
Ohtsuka, N., M. Nakano and H. Ueyama, 1981. Acoustic emission monitoring during rupture test of pressure vessel and laboratory fracture test. J. Pressure Vessel Technol., 103(2): 191-199.

Pan, R.B., Y. Fan and X.L. Yang, 2004. Nondestructive testing of pressure vessels: Ultrasonic testing technology (II). NDT, 26(5): 244-248, (In Chinese).

Rastogi, P.K., 1997. Optical Measurement Techniques and Applications. Artech House Inc., Boston, pp: 183-197.

Sciammarella, C.A., 1982. The moire method: A review. Exp. Mech., pp: 418-433.

Sha, F.H. and X. Chen, 1994. The application of moiré interferometry to fracture analysis of pressure vessels. J. Taiyuan Univer. Technol., 25(1): 58-64, (In Chinese).

Shen, G.T. and W.L. Zhang, 2004. A review of nondestructive testing techniques for pressure vessels. NDT, 26(1): 37-40, (In Chinese).

Sposito, G., C. Ward, P. Cawley, P.B. Nagy and C. Scruby, 2010. A review of non-destructive techniques for the detection of creep damage in power plant steels. NDT E Inter., 43: 555-567.
Taylor, D.J., T.R. Watkins, C.R. Hubbard and M.R. Hill, 2012. Residual stress measurements of explosively clad cylindrical pressure vessels. J. Pressure Vessel Technol., 134: 1-8.

Yao, X.F., L.B. Meng, J.C. Jin and H.Y. Yeh, 2005. Full-field deformation measurement of fiber composite pressure vessel using digital speckle correlation method. Polym. Test, 24: 245-251.

Yue, L., R.M. Tian and X.H. Han, 1995. Determination of subsidiary bending stresses at the place of angular distortion and the misaligned contact edge of the pressure vessels by ELM. J. Exp. Mech., 10(3): 228-235, (In Chinese).

Zhang, Y.X., 2005. Design and reliablity analysis for a new type of high-pressure quick-opening structure. Ph.D. Thesis, Beijing University of Chemical Technology, (In Chinese).

Zhou, Y.F. and G.T. Shen, 2005. Nondestructive testing of pressure vessels: Nondestructive testing technology for ultra-high pressure vessels. NDT, 27(4): 202-207, (In Chinese). 